\title{
Confocal Microwave Imaging for Breast Cancer Detection: Localization of Tumors in Three Dimensions
}

\author{
Elise C. Fear*, Member, IEEE, Xu Li, Student Member, IEEE, Susan C. Hagness, Member, IEEE, and \\ Maria A. Stuchly, Fellow, IEEE
}

\begin{abstract}
The physical basis for breast tumor detection with microwave imaging is the contrast in dielectric properties of normal and malignant breast tissues. Confocal microwave imaging involves illuminating the breast with an ultra-wideband pulse from a number of antenna locations, then synthetically focusing reflections from the breast. The detection of malignant tumors is achieved by the coherent addition of returns from these strongly scattering objects. In this paper, we demonstrate the feasibility of detecting and localizing small $(<1 \mathrm{~cm})$ tumors in three dimensions with numerical models of two system configurations involving synthetic cylindrical and planar antenna arrays. Image formation algorithms are developed to enhance tumor responses and reduce early- and late-time clutter. The early-time clutter consists of the incident pulse and reflections from the skin, while the late-time clutter is primarily due to the heterogeneity of breast tissue. Successful detection of 6-mm-diameter spherical tumors is achieved with both planar and cylindrical systems, and similar performance measures are obtained. The influences of the synthetic array size and position relative to the tumor are also explored.
\end{abstract}

Index Terms-Cancer, image reconstruction, microwave imaging, object detection.

\section{INTRODUCTION}

$\mathbf{X}$ -RAY mammography is currently the most effective imaging method for detecting nonpalpable early-stage breast cancer [1]. However, despite significant progress in improving mammographic techniques for detecting and characterizing breast lesions, persisting limitations related to sensitivity and specificity result in a relatively high false-negative rate $(4 \%-34 \%)$ [2] and high false-positive rate (70\%) [3], particularly in patients with radiographically dense breast tissue [4]. These difficulties arise from the small (few percent) intrinsic contrast between diseased and normal tissue at X-ray frequencies. Breast compression is required to reduce image blurring and to create uniformity of tissue between the source

Manuscript received August 3, 2001; revised March 8, 2002. The work of E. C. Fear and M. A. Stuchly was supported by the Natural Sciences and Engineering Research Council of Canada. The work of X. Li and S. C. Hagness was supported by The Whitaker Foundation under Biomedical Engineering Research Grant RG-99-0004. Asterisk indicates corresponding author.

*E. C. Fear is with the University of Calgary, Electrical and Computer Engineering, 2500 University Drive NW, Calgary, AB T2N 14N Canada (e-mail: fear@enel.ucalgary.ca).

X. Li and S. C. Hagness are with the University of Wisconsin-Madison, Electrical and Computer Engineering, Madison, WI 53706-1691 USA.

M. A. Stuchly is with the University of Victoria, Electrical and Computer

Engineering, STN CSC, Victoria, BC, V8W 3P6, Canada.

Publisher Item Identifier 10.1109/TBME.2002.800759. and receiver located on opposing sides of the breast. The association of X-ray mammography with uncomfortable or painful breast compression and exposure to low levels of ionizing radiation may reduce patient compliance with screening recommendations. These concerns motivate the search for techniques that image other physical tissue properties or metabolic changes [5], [6].

The motivation for developing a microwave imaging technique for detecting breast cancer is the significant contrast in the dielectric properties at microwave frequencies of normal and malignant breast tissue suggested by published measured data [7]-[9], and by our own preliminary measurements on freshly excised breast tissue [10]. The estimated malignant-to-normal breast tissue contrast is between $2: 1$ and $10: 1$, depending on the density of the normal tissue. Thus, while microwave technology does not offer the potential for the high spatial resolution provided by X-rays, it does offer exceptionally high contrast with respect to physical or physiological factors of clinical interest, such as water content, vascularization/angiogenesis, blood-flow rate, and temperature. Furthermore, microwave attenuation in normal breast tissue is low enough to make signal propagation through even large breast volumes quite feasible. Microwave imaging techniques result in a three-dimensional (3-D) volumetric map of the relevant tissue properties rather than a two-dimensional (2-D) projection. The combination of these features eliminates the need for breast compression. In addition, microwave technology would be nonionizing and noninvasive. For these reasons, microwave breast imaging has the potential to overcome some of the limitations of conventional breast cancer screening modalities.

There is a growing body of literature on medical applications of microwave imaging [11]. Two different classes of active microwave imaging techniques exploit contrasts in dielectric properties: tomographic methods and backscatter methods. The goal of microwave tomography is the recovery of the dielectric-properties profile of an object from measurements of the microwave energy transmitted through the object (for example, see [12]-[16]). Promising initial experimental results for breast imaging have been obtained recently [17], [18]. The challenge of microwave tomography, however, is that it involves the solution of an ill-conditioned nonlinear inversescattering problem. As a result, these approaches are inherently limited by vulnerability to small experimental uncertainties and noise. Furthermore, the inverse-scattering problem requires image reconstruction algorithms that may be computationally intensive. 
Analogous to ground penetrating radar [19], backscatter methods use the measured reflected signals to infer the locations of significant microwave scatterers. Scattering arises from significant contrasts in dielectric properties, for example, between normal breast tissue and malignant lesions. Initially, active microwave backscatter techniques for breast cancer detection [20]-[22] were unsuccessful because a single antenna location was used for transmitting and receiving, eliminating the possibility of spatially focusing the signal. Without the spatial selectivity obtained by focusing, the tumor signature can be easily masked by clutter from adjacent breast regions. Hagness et al. recently proposed a confocal microwave imaging (CMI) approach to breast cancer detection [23]-[26]. CMI involves illuminating the breast with an ultra-wideband pulse from a number of physical antenna locations. The relative arrival times and amplitudes of the backscattered signal provide information that is used to determine the scatterer location using simple, robust synthetic focusing techniques. In contrast to microwave tomography, the CMI approach [23]-[29] seeks only to identify the presence and location of strong scatterers in the breast rather than attempting to completely reconstruct the dielectric-properties profile.

Preliminary investigations of CMI for breast imaging in two dimensions have shown that simple focusing algorithms are robust with respect to realistic dielectric-properties variations [23], [26], [28], dispersion [23], [26], and complex anatomical structure [26] of normal breast tissue. Although the initial 2-D results appear promising, a number of issues must be examined to evaluate the suitability of CMI for clinical use. In this paper, we demonstrate through numerical simulations the feasibility of using two different CMI system configurations to detect and to localize small tumors in three dimensions. In Section II, we first describe the breast models and simulation methods used to obtain representative backscatter waveforms. The use of simple breast models provides insight into the influence of various performance parameters, such as the number of antennas required for successful detection, and allows for comparison of results obtained with two different system configurations, cylindrical and planar. Second, we present CMI reconstruction algorithms that have been synthesized for 3-D applications. Third, we describe the measures used to evaluate images constructed from the backscatter waveforms. Results demonstrating the capabilities of both configurations to detect and to localize tumors in three dimensions are presented and discussed in Section III. Conclusions are summarized in Section IV.

\section{Methods}

Scanning the breast for tumors with CMI involves collecting backscatter from the breast after illumination with an ultra-wideband signal. The backscatter is processed and synthetically focused at points in the domain of interest. In this paper, backscatter data are computed using the finite-difference time-domain (FDTD) method [30] and simple breast models. This approach allows for rapid development and evaluation of image reconstruction algorithms and CMI system designs.

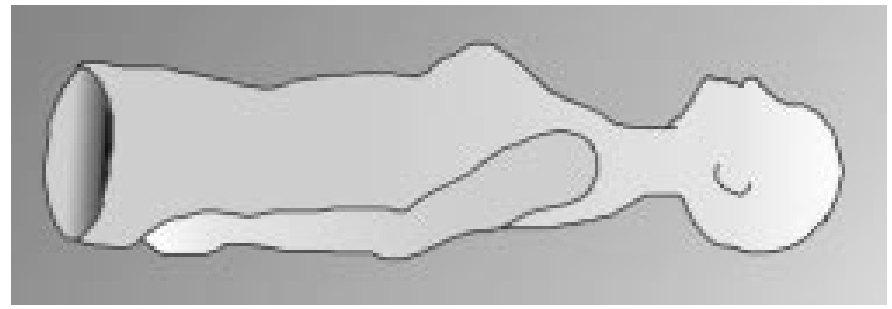

(a)

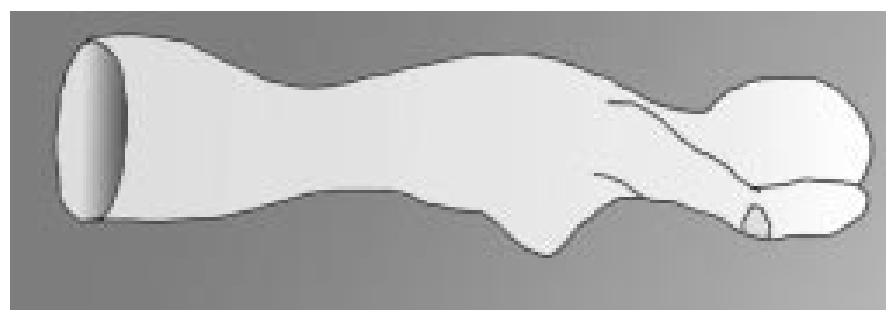

(b)

Fig. 1. Patient orientation for the (a) planar and (b) cylindrical systems.

\section{A. Models}

We are investigating planar [23]-[26] and cylindrical [27]-[29] systems for breast tumor detection with CMI. The patient orientation is different for the planar and cylindrical system configurations. In the planar configuration, the patient is oriented in a supine position [Fig. 1(a)] and a resistively loaded bowtie antenna [24], [25] is scanned over the surface of the naturally flattened breast to create a synthetic planar array [Fig. 2(a)]. With the cylindrical configuration, the patient is oriented in a prone position with the breast naturally extending through a hole in the examination table [Fig. 1(b)]. A resistively loaded dipole antenna is scanned around the breast to create a synthetic cylindrical array [27], [28] [Fig. 2(b)]. The difference in patient orientation leads to the development of simple breast models appropriate for each configuration. With the planar system, the breast is modeled as a half-space of heterogeneous breast tissue bounded by a 2-mm-thick layer of skin [Fig. 3(a)]. For the cylindrical system, the breast model is a 6.8 -cm-diameter cylinder surrounded by a 2 -mm-thick layer of skin [Fig. 3(b)]. With both systems, perfectly matched layer absorbing boundary conditions [31] are used to terminate the computational domain. Consequently, the cylindrical model has infinite length in the $z$ direction and the planar model has infinite extent in the $x-y$ plane. To connect our simple models to the patient orientation, we define standard imaging planes as indicated in Fig. 4. Although the breast models differ in shape, an equivalent imaging task is simulated with each system, specifically the detection and localization of a 6-mm-diameter tumor located a minimum distance of $3 \mathrm{~cm}$ below the skin.

The materials in the simple breast models [indicated in Fig. 3(a) and (b)] are assigned appropriate electrical properties. The dielectric constant and conductivity for malignant breast tissue are assumed to be $\varepsilon_{r}=50$ and $\sigma=4 \mathrm{~S} / \mathrm{m}$, respectively, over the microwave frequency band. The dielectric properties of normal breast tissue are assigned random variations of up to $\pm 10 \%$ around nominal values of $\varepsilon_{r}=9$ and $\sigma=0.4$ $\mathrm{S} / \mathrm{m}$, distributed over $4-\mathrm{mm}$ cubes. Thus, the assumed contrast between malignant and normal breast tissue is approximately 


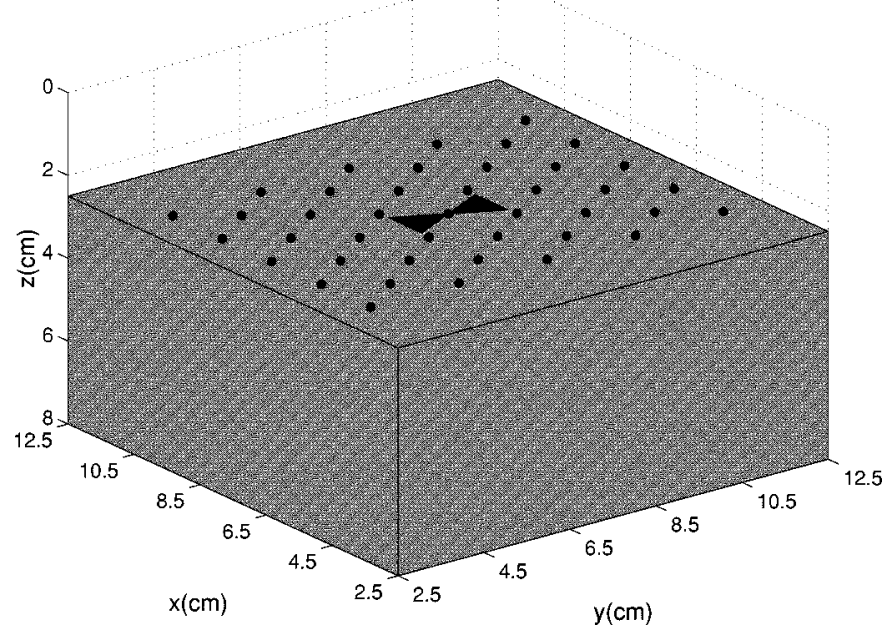

(a)

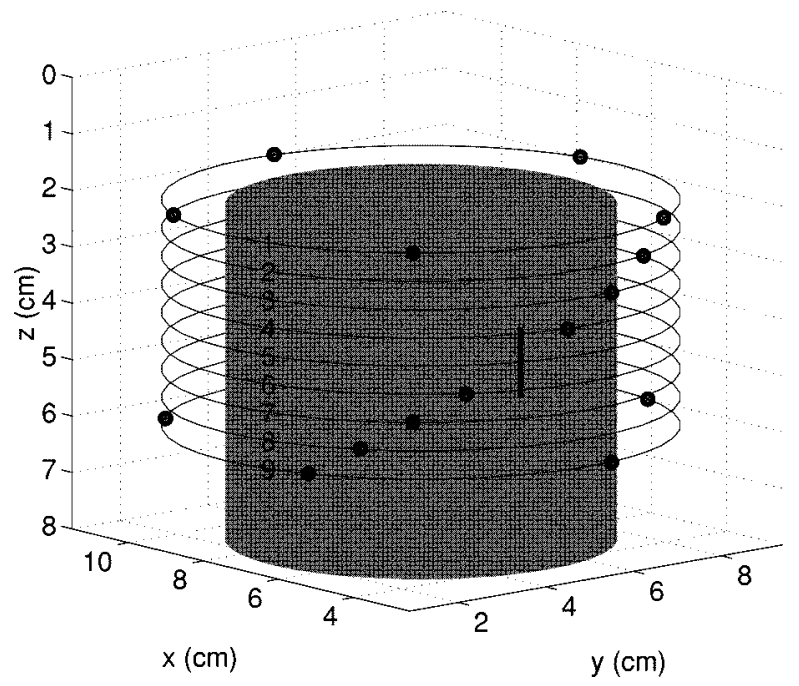

(b)

Fig. 2. Arrangement of antenna array for (a) planar and (b) cylindrical systems. The small circles show where the antenna is repositioned to create the synthetic array. Fig. 2(b) shows all antennas in row 1 and four antennas in row 9. Selected antennas in rows $2-8$ are included to demonstrate the staggered arrangement.

$5: 1$ in relative permittivity and $10: 1$ in conductivity. Similar to our previous heterogeneous breast tissue models [23], the $\pm 10 \%$ variations represent the expected variations in real breast tissues. This range is suggested by measurement studies reported by Joines et al. [8] and Chaudhary et al. [9], and is assumed to result from the presence of tissues ranging from fat to fibroglandular. The skin is assigned the following values: $\varepsilon_{r}=36$ and $\sigma=4 \mathrm{~S} / \mathrm{m}$. Since we had previously shown that the CMI approach was robust with respect to pulse broadening effects caused by the dispersive properties of biological tissue [23], [26], we neglected the frequency-dependent properties in our current 3-D models.

One antenna is used to scan the breast at the various locations, as shown in Fig. 2(a) and (b). The bowtie antenna, which is placed directly on the skin, is moved to 41 locations arranged in five rows of five positions each interleaved with four rows of four positions each [Fig. 2(a)]. The size of the bowtie antenna used here is $2 \mathrm{~cm}$ in length (a smaller version of that presented

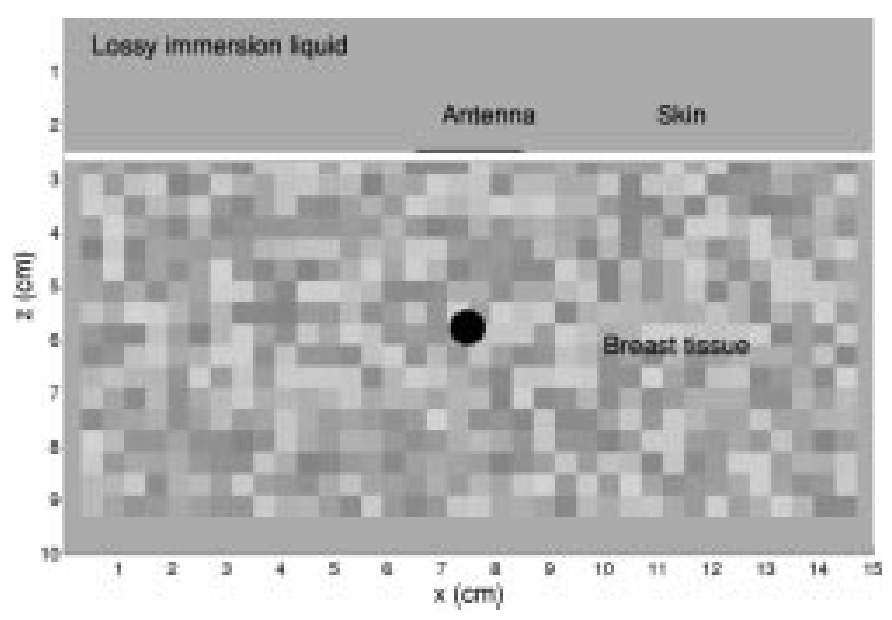

(a)

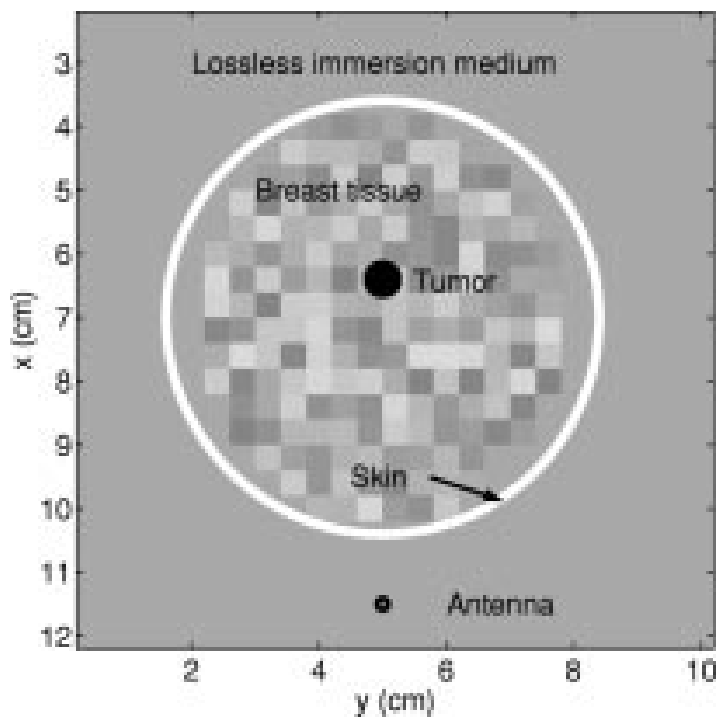

(b)

Fig. 3. Model geometry for the (a) planar system (side view), where the antenna is backed by an impedance matching layer of lossy liquid similar to breast tissue, and (b) cylindrical system (top view), where the breast and antennas are immersed in a lossless material similar to breast tissue.

in [25]). The synthetic planar array spans $6 \mathrm{~cm}$ in the $x$ direction [defined as the distance between the antenna feed locations marked by the small circles in Fig. 2(a)] and $8.2 \mathrm{~cm}$ in the $y$ direction. The dipole antenna, which is located at a distance of 1 $\mathrm{cm}$ from the surface of the breast, is moved to 45 locations arranged in nine rows of five staggered positions each [Fig. 2(b)]. The dipole antenna is $1.25 \mathrm{~cm}$ in length, and the synthetic cylindrical array spans $4 \mathrm{~cm}$ along the $z$ axis [defined as the distance between antenna feed locations marked by the small circles in Fig. 2(b)]. For both systems, synthetic arrays with only 25 elements are also considered. In this case, the five rows of five antenna positions in Fig. 2(a) are used for the planar system and the odd numbered rows in Fig. 2(b) are used for the cylindrical system. For comparison of the two systems, the synthetic arrays are centered over a 6-mm-diameter tumor, which is located at least $3 \mathrm{~cm}$ from the antennas. To examine the impact of arrays with fewer elements and tumors offset from the axial center of the array, additional subsets of the cylindrical synthetic array are also considered. 
At each antenna position, the bowtie or dipole antenna is excited with an ultra-wideband pulse of the form

$$
V(t)=V_{o}\left(t-t_{o}\right) e^{-\left(t-t_{o}\right)^{2} / \tau^{2}}
$$

where $V_{o}$ is used to adjust the amplitude of the pulse, $\tau=62.5$ ps and $t_{o}=4 \tau$. This pulse has full-width at half-maximum (FWHM) of $0.17 \mathrm{~ns}$ in time and approximately $6 \mathrm{GHz}$ in frequency, with maximum content near $4 \mathrm{GHz}$. During and following excitation, the current (planar configuration) or voltage (cylindrical configuration) at the antenna feed is recorded. The recorded signals contain the backscatter from the breast.

\section{B. Image Formation}

The recorded signals have early and late time content. The early-time content is dominated by the incident pulse, reflections from the skin and residual antenna reverberations. The late-time content contains tumor backscatter and backscatter due to clutter. The signal processing goals are to reduce the early-time content, which is of a much greater amplitude than the tumor response, and to selectively enhance the tumor response while suppressing the clutter to permit reliable detection of tumors in the reconstructed images. Using a straightforward algorithm, images are reconstructed with the postprocessed signals. The image formation steps are described below.

1) Calibration: The goal of the calibration step is to remove the incident pulse and skin backscatter from the recorded waveforms. Calibration procedures are based on the assumption that the signals recorded at various antenna locations have similar incident pulse and skin backscatter content. For the planar system, the calibration signal is formed as the average of the returns at each antenna in a given row. Subtraction of this signal from the actual response recorded at each antenna in that row suppresses the early-time content. For the cylindrical system, antennas are located at a distance from the skin, so the incident pulse and skin returns are separated in time. Subtracting returns recorded at each antenna location without the breast model present reduces the incident pulse. The skin reflection is the dominant component of the resulting signals. Because antennas may be located at slightly different (submillimeter) distances from the skin, alignment of skin reflections is required for effective reduction with calibration [29]. The calibration is completed by subtracting the average of the aligned signals in a given row from each return in that row.

2) Integration: The next step in the signal processing is the integration of the calibrated signals. The differentiated Gaussian excitation signal has a zero-crossing at its center point in time. The backscattered signal that would follow after a specific time delay corresponding to the round-trip distance between the antenna and the scatterer would also have a zero-crossing at its center point. After integration, the signal would have a maximum at the center point, allowing for the coherent addition of local maxima via straightforward time-shifting. This process of synthetic focusing, which serves as the rationale for integration, is discussed further in Step 4) below.

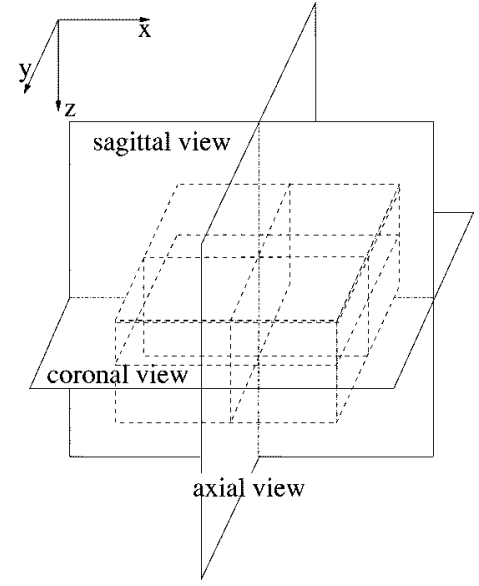

(a)

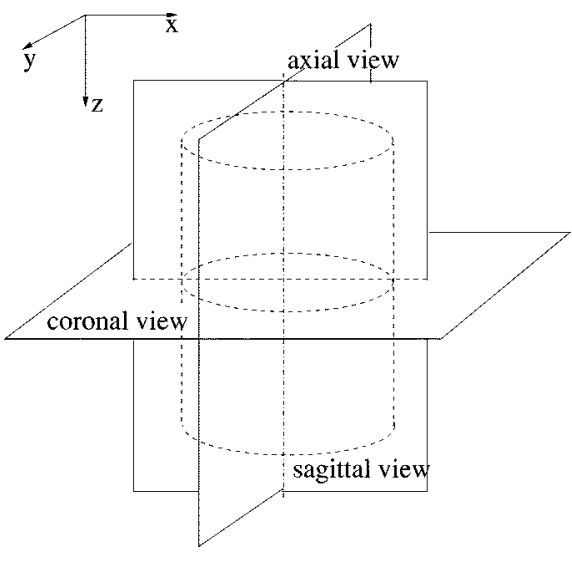

(b)

Fig. 4. Image planes for the (a) planar and (b) cylindrical systems.

3) Compensation: Compensation for radial spreading and/or path loss is applied to the signals. Radial spreading correction accounts for the decrease in amplitude of a spherical wave as it expands, while path loss compensation corrects for the reduction in signal strength due to propagation through lossy breast tissue. To estimate these compensation factors for the planar system, a bowtie antenna immersed in homogeneous lossy breast tissue is excited with the signal in (1) and simulated with FDTD. Field amplitudes are computed at points along a line perpendicular to the antenna and passing through the feed. The results are linearly interpolated to provide estimates of the total spreading and loss at required distances from the antenna. With the cylindrical system, radial spreading is estimated with a $1 / r$ model, where $r$ is the distance from the antenna. Simulations confirm that this approach provides reasonable estimates of radial spread. Path loss compensation is neglected in the cylindrical configuration, as it enhances clutter that does not originate inside of the breast. For both systems, the propagation distance corresponding to each time step in the recorded, processed waveforms is calculated and the appropriate compensation factors are applied.

4) Image Reconstruction: To form the image, the processed signals are synthetically focused at a specific point in the breast. First, distances from each antenna to the focal point are computed and converted into time delays. The time delays are used 


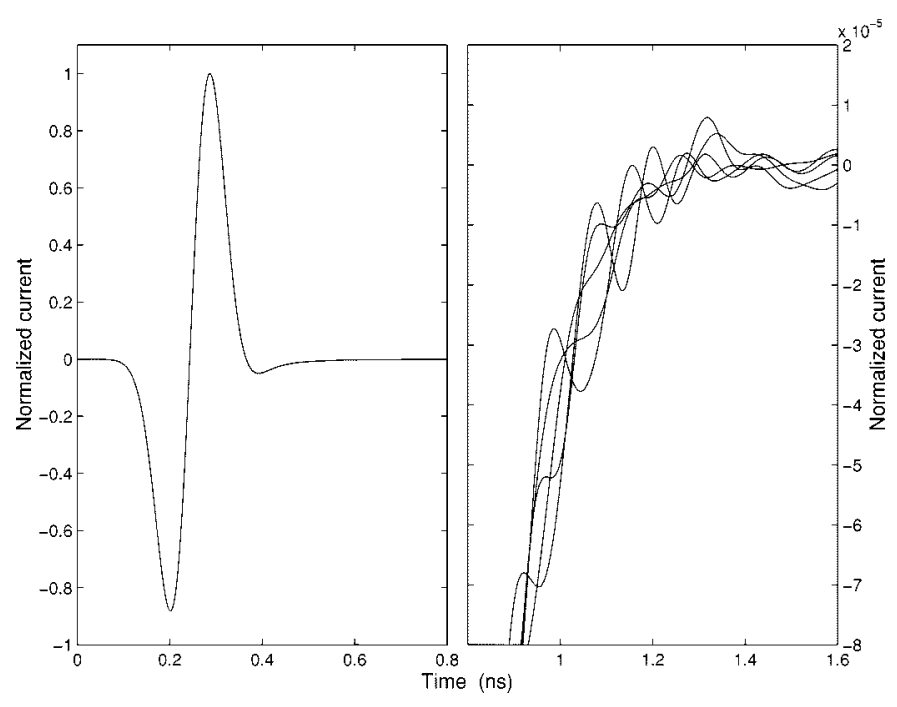

(a)

(b)

Fig. 5. The recorded signals at a selected row of antennas in the planar system. (a) Early-time response. (b) Late-time response.

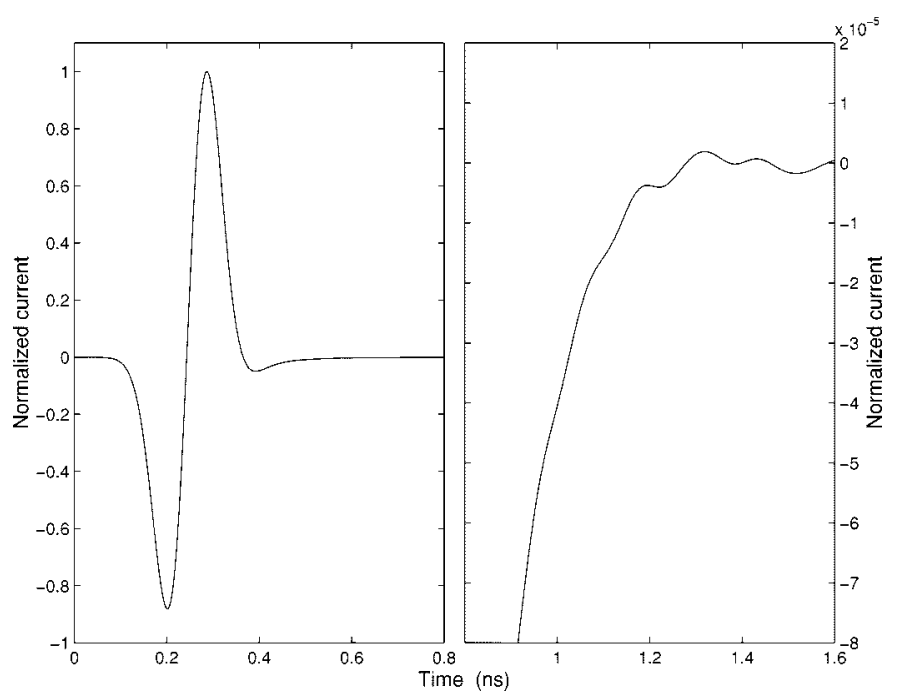

(a)

(b)

Fig. 6. The calibration signal for the selected row of antennas in the planar system. (a) Early-time response. (b) Late-time response.

to identify the contribution from each processed signal. All contributions are summed and the squared value of this sum is assigned to the pixel value at the focal point. The intensity value $(I)$ of the pixel at the location $\vec{r}$ is assigned as

$$
I(\vec{r})=\left[\sum_{m=1}^{M} B_{m}\left(\tau_{m}(\vec{r})\right)\right]^{2}
$$

where $B_{m}$ is the postprocessed backscatter waveform at the $m$ th antenna located at $\vec{r}_{m}$ and $\tau_{m}(\vec{r})=2\left|\vec{r}-\vec{r}_{m}\right| /(v \Delta t)$ is the discrete time delay from the $m$ th antenna to the synthetic focal point at $\vec{r}$. Here, $v$ is the assumed velocity of propagation of the signal in the medium, calculated by assuming that the breast tissue is homogeneous $\left(\varepsilon_{r}=9\right)$. The focal point is scanned to a new location in the 3-D region of interest, and this process is repeated. For both systems, the focal point coordinates are defined with respect to the location of the skin. With the planar system, the focal point locations extend from the lower boundary of the skin into the breast interior. With the cylindrical system, estimates of the skin location [29] bound the focal points.

\section{Performance Measures}

1) Single-Signal Analysis: To examine the influence of each step in the signal processing sequence, the backscatter recorded at a single antenna is studied in detail. To follow the enhancement of the tumor response during the image formation process, we compare the peak-to-peak tumor response with that of the total signal at each of the four steps. Estimation of the peak-to-peak tumor response requires additional simulations. The tumor is removed from the breast model, and backscatter is recorded at each antenna in the selected row. We isolate the tumor response by subtracting the tumor-free returns recorded at each antenna location from the recorded signals for the model containing a tumor. The average tumor response for the selected row of antennas is computed, and used to calibrate the tumor responses recorded at each antenna in the row. The peak-to-peak of the tumor response is calculated initially and after each signal processing step. These results are compared with those calculated for the total signal.

2) Images: To investigate tumor detection, images are formed for the tumor-bearing breast model and the tumor-free model. Comparison of results ensures that tumors are detected only when present in the models. The influence of the number of antennas is examined by comparing images reconstructed with the full set of antenna positions (41 or 45) and the limited set (25 antenna positions). The results of changing array span and tumor location are also examined. Several measures related to the tumor response and clutter are defined to evaluate the images. To investigate tumor localization, the location and FWHM extent of the tumor response are determined. Two signal-to-clutter (SC) ratios are defined to quantify the magnitude of the tumor response. The within-breast $\mathrm{SC}$ ratio compares the maximum tumor response with the maximum clutter response in the same image. The maximum clutter is determined by locating the maximum pixel value outside of the volume containing the imaged tumor (defined by twice the extent of the FWHM response of the tumor). The between-breast $\mathrm{SC}$ ratio compares the maximum tumor response with the pixel intensity at the same location in the image formed without a tumor present. To provide additional insight into the clutter behavior, the mean and standard deviation of the clutter are computed.

\section{RESULTS AND DisCUSSION}

\section{A. Single-Signal Analysis}

To demonstrate the influence of signal processing steps 1 , 2 , and 3 , signals at the central antenna and corresponding row in the planar configuration [Fig. 2(a)] are examined in detail. Fig. 5 shows the signals recorded by the selected row of antennas in the planar array. The early-time responses, which include the incident pulse and skin reflection, overlap for all of these antenna locations [Fig. 5(a)]. The clutter and tumor response are buried in the late-time response, which are shown in smaller scale [Fig. 5(b)]. The calibration signal, shown in Fig. 6, 


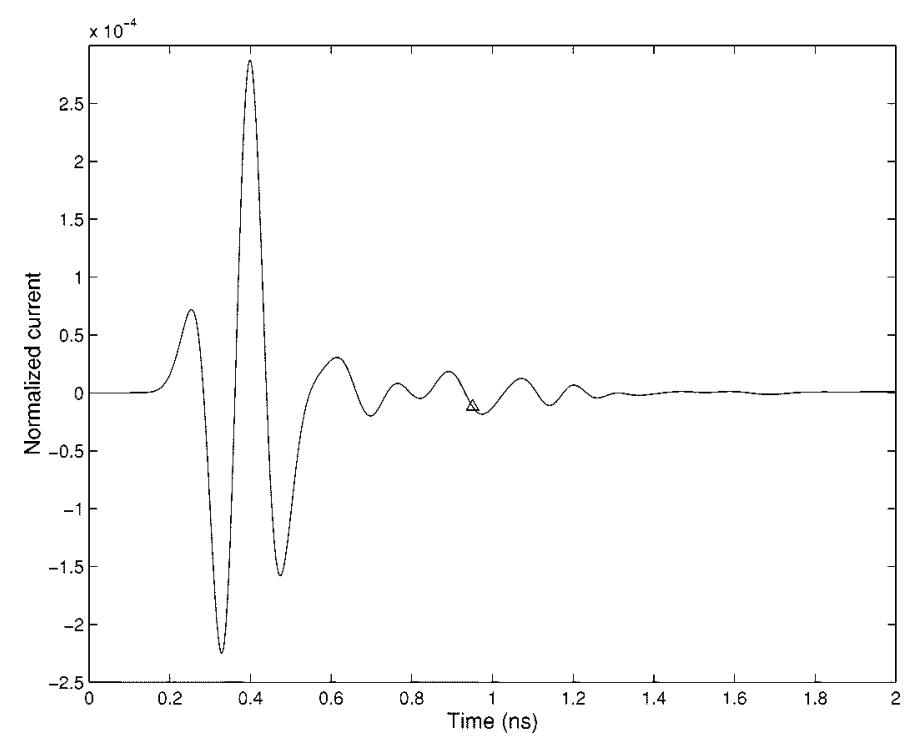

Fig. 7. Calibrated signal at the center antenna of the planar system [Step 1]. The triangle marks the location of the tumor response.

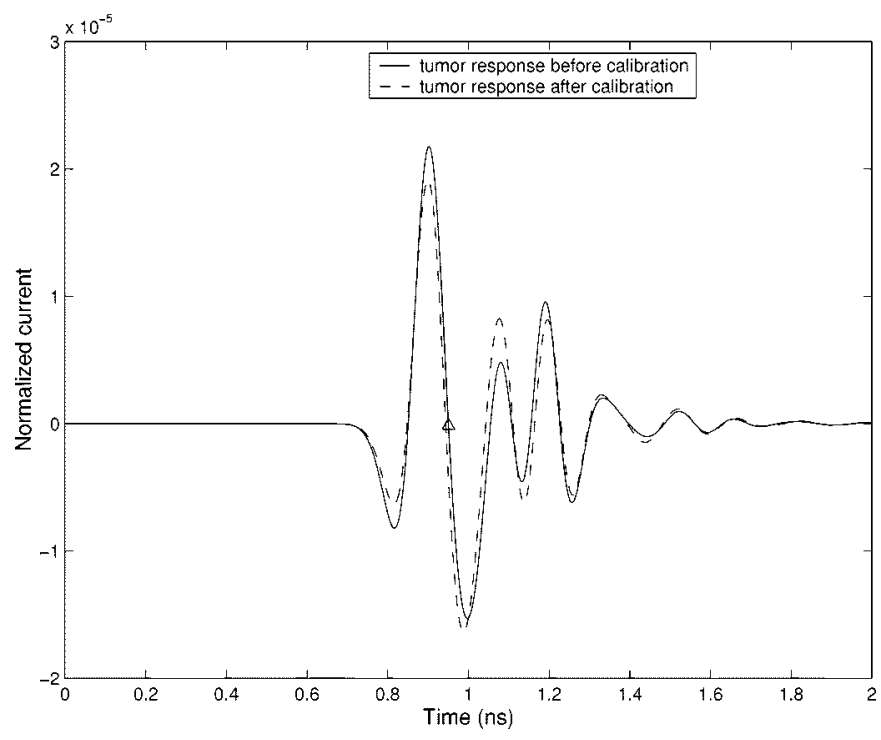

Fig. 8. Tumor response before and after calibration (planar system).

is calculated by averaging the returns recorded at all antennas in this row. The early-time response in the calibration signal represents the incident pulse and skin reflection [Fig. 6(a)]. Because the tumor and clutter responses in Fig. 5(b) add incoherently, the late-time calibration signal [Fig. 6(b)] represents an estimate of the late-time incident pulse decay and skin reflection. The vertical scale of Fig. 7, which shows the signal after calibration [Step 1] for the antenna located at the center of the array, illustrates that the incident pulse is greatly reduced in signal strength. Clutter, due to incomplete skin cancellation, breast tissue heterogeneity, and other sources, is also clearly evident. The tumor response, the location of which is indicated by the triangle symbol, is buried in the clutter and not readily apparent in Fig. 7. For comparison only, the initial and calibrated tumor responses are obtained using the tumor-free model (as described in Section II-C) and are presented in Fig. 8. It is evident from Fig. 8 that calibration has negligible influence on the tumor response. Therefore, calibration accomplishes the goal

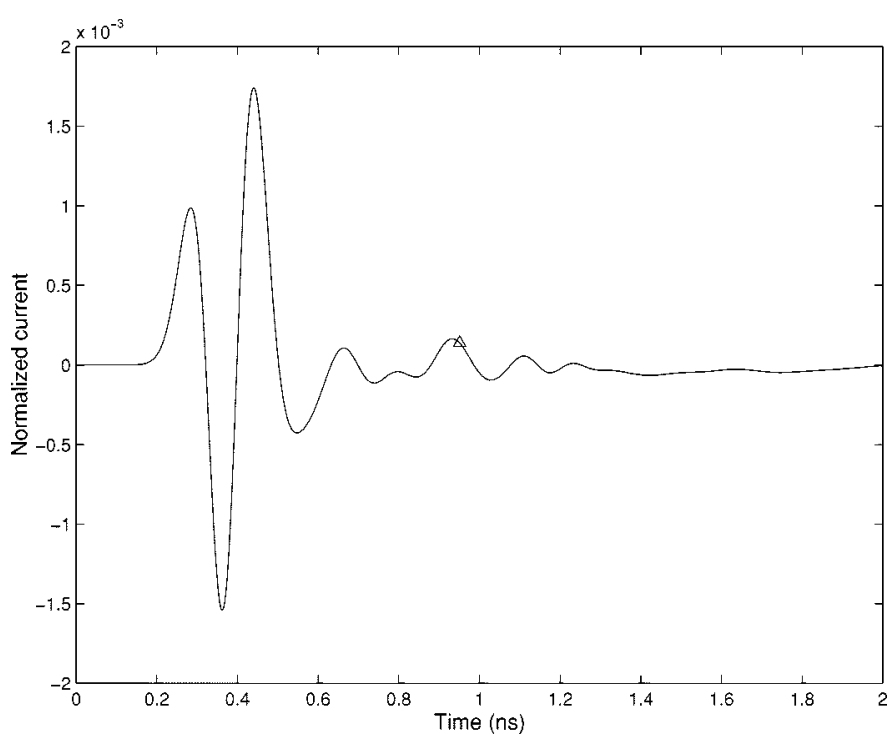

Fig. 9. Calibrated signal at center antenna of planar system, after integration [Step 2]. The triangle marks the tumor response.

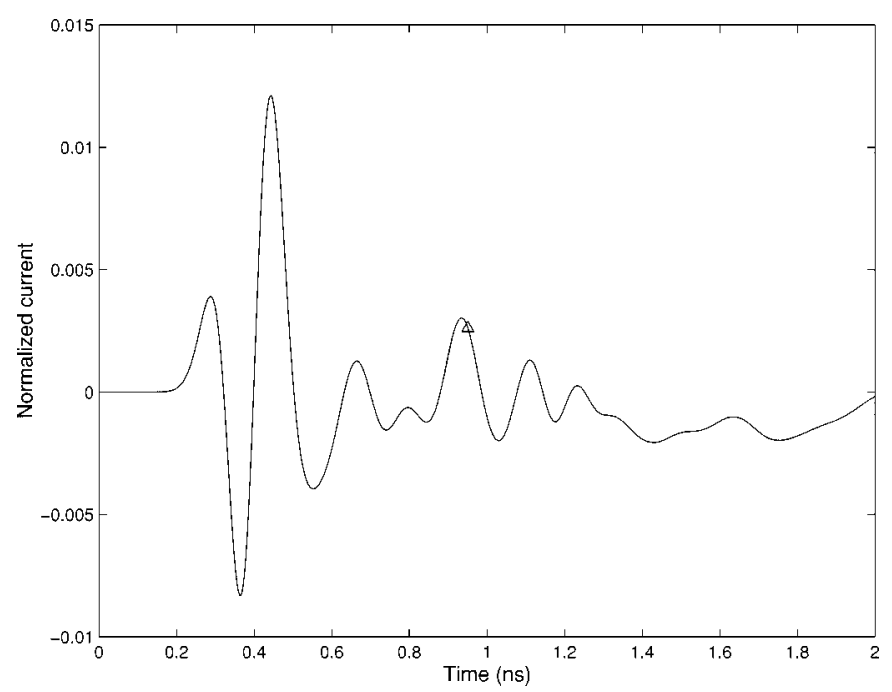

Fig. 10. Signal at center antenna in planar system, after compensation [Step 3].

TABLE I

Ratios BetweEn PEAK-TO-PEAK TUMOR RESPONSE AND TOTAL SignAL FOR ANTENNA ClOSEST TO TUMOR

\begin{tabular}{|c|c|c|}
\hline \multirow[t]{2}{*}{ Stage } & \multicolumn{2}{|c|}{$\begin{array}{l}\text { Peak-to-peak tumor/total signal } \\
\qquad(\mathrm{dB})\end{array}$} \\
\hline & Cylindrical & Planar \\
\hline Initial & -99.6 & -94.1 \\
\hline Calibration & -20.1 & -22.8 \\
\hline Integration & -23.0 & -20.5 \\
\hline Compensation & -7.0 & -11.4 \\
\hline
\end{tabular}

of reducing early-time content without degrading the tumor response. The signal after integration [Step 2] is shown in Fig. 9, while Fig. 10 shows the signal after compensation [Step 3]. Comparison of Figs. 7 and 9 shows that the tumor response occurs at a local maximum after integration. Comparison of Figs. 9 and 10 illustrates the significant tumor enhancement contributed by the compensation step. 
To provide measures of the relative tumor enhancement at each signal processing step, the ratios between the peak-to-peak responses of the tumor and total signal are summarized in Table I for both systems. The ratios computed for the original signals provide estimates of the dynamic range required in a practical system. The cylindrical system requires a greater dynamic range, as the antenna-tumor separation is greater than in the planar system. The ratios computed after calibration illustrate the reduction in early-time response achieved with this step. Compensation significantly improves the relative tumor response, however, the tumor cannot be readily identified and localized with this single signal. Synthetic focusing of multiple antenna returns achieves the suppression of clutter and enhancement of the tumor response needed for robust tumor detection.

\section{B. Images}

Synthetic focusing is applied to the processed data, and the resulting images are shown for the three orthogonal planes defined in Fig. 4(a) (planar system) and 4(b) (cylindrical system). Images of tumor-bearing breast models are presented in Figs. 11 (planar) and 12 (cylindrical). Images of tumor-free breast models are shown in Figs. 13 (planar) and 14 (cylindrical). Figs. 11 and 13 are normalized to the maximum of the reconstructed planar data set for the tumor-bearing model. Figs. 12 and 14 are normalized to the maximum of the reconstructed cylindrical data set for the tumor-bearing model. The tumor is easily detected in Figs. 11 and 12, illustrating the enhancement of the tumor response via coherent addition of returns from the scattering object (the malignant tumor). Also evident in these images is the localization of the tumor in three dimensions. Images of tumor-free breast models (Figs. 13 and 14) show no evidence of strong scatterers. Rather, the pixel values correspond simply to clutter. This illustrates the process of incoherent addition of returns from spatially distributed heterogeneities in normal breast tissue. To further evaluate the performance of CMI, the location and FWHM size of the tumor responses, as well as SC ratios, are computed for Figs. 11-14 and summarized in Table II.

The SC ratios further demonstrate the ability of synthetic focusing to provide reliable tumor detection, and also suggest a method for false-alarm reduction. Estimates of the within-breast $\mathrm{SC}$ ratio before focusing are provided by the peak-to-peak ratios of the tumor to the total signal summarized in Table I. Comparing the within-breast SC ratios in Table II with the ratios calculated after compensation shows that gains of approximately $11 \mathrm{~dB}$ (cylindrical) and $15 \mathrm{~dB}$ (planar) are achieved with synthetic focusing. The within-breast $\mathrm{SC}$ ratios are similar for planar and cylindrical configurations, indicating that the tumor is easily detected with both systems. The between-breast SC ratios demonstrate the significant difference in images obtained for the tumor-free and tumor-bearing models. Further, these results suggest that comparing images of the left and right breast may further assist in tumor detection. For example, assume that the left breast is represented by Fig. 12(c), and the right by Fig. 14. Comparison of pixel values suggests the presence of a tumor near the center of the left breast. A smaller response is located near $(7 \mathrm{~cm}, 7 \mathrm{~cm})$ in both images, suggesting that this is

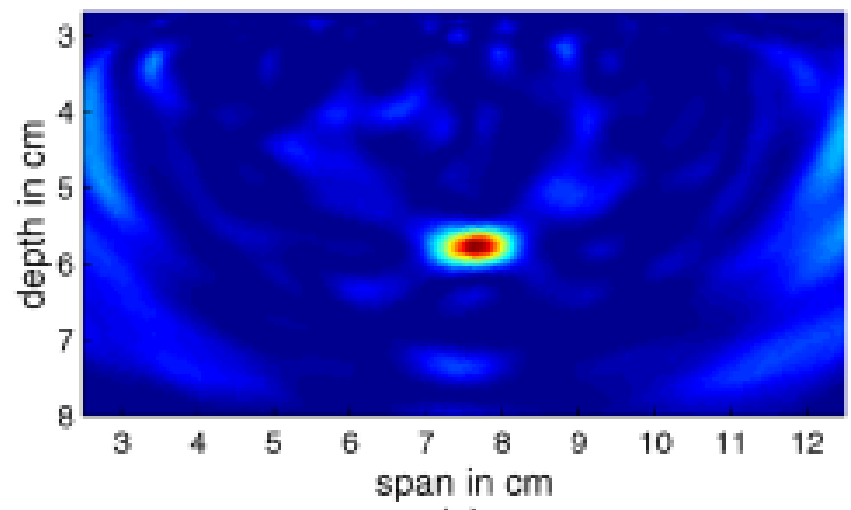

(a)

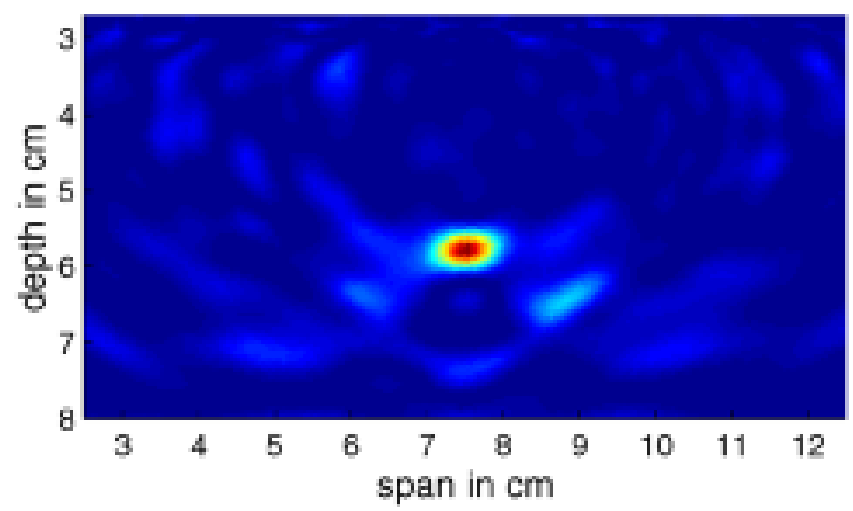

(b)

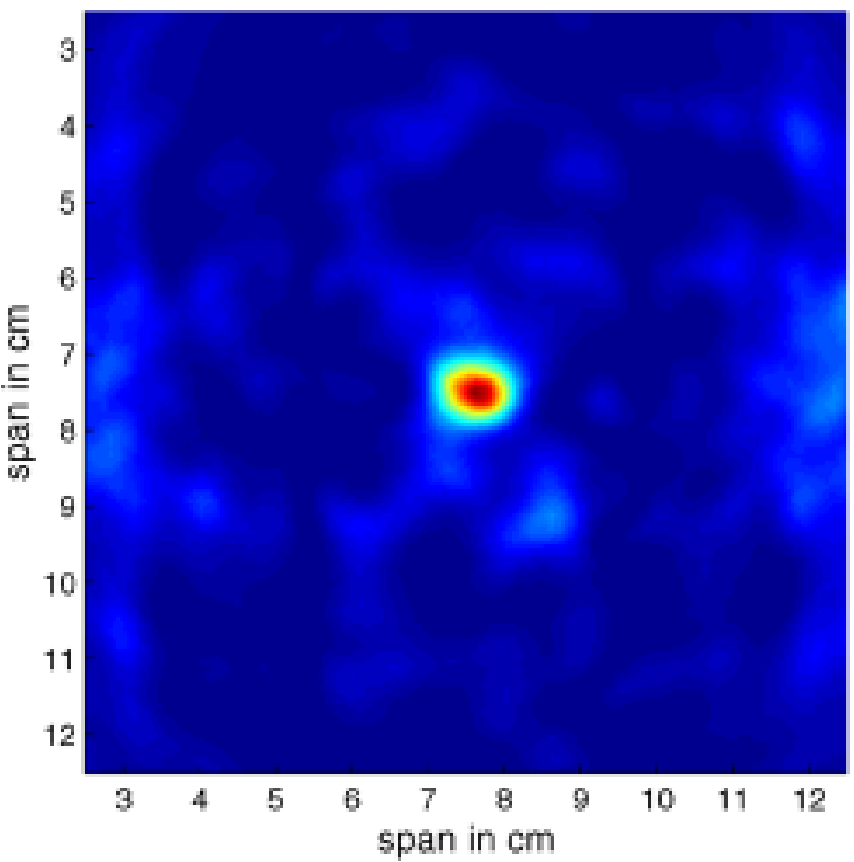

(c)

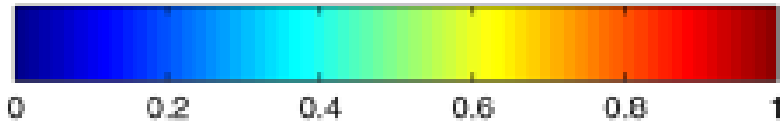

Fig. 11. Three-dimensional reconstructed image, shown in three planes, for the planar system: (a) axial, (b) sagittal, and (c) coronal. The scale for (a)-(c) is shown below (c). 
clutter arising from common features rather than a tumor. Due to the similarities in the density and heterogeneity of the right and left breasts (and, therefore, similar clutter signal levels), this approach is expected to aid in distinguishing tumor responses from clutter, hence reducing false alarms.

To further investigate the influence of the number of antennas on tumor detection, images are reconstructed with fewer antennas ( 25 instead of 41 or 45 ) and corresponding measures are summarized in Table III. The tumor response remains evident in images, and similar localization and FWHM extents are obtained. However, the within-breast $\mathrm{SC}$ ratios decrease due to a relative increase in clutter. The clutter variance increases with fewer antennas, as expected. We note that for the cylindrical system, the between-breast SC ratio unexpectedly improves. In this case, the incoherent addition of returns happens to result in a smaller pixel value for the tumor-free image at the desired location. Although detection of the 6-mm diameter tumor is maintained, smaller and deeper tumors are expected to have smaller responses [25], and may be obscured by clutter in the final images. With the cylindrical system, larger breast dimensions than those used in this study are anticipated. The number of antennas required to scan a larger breast model is expected to increase, as reliable detection requires sufficient spatial sampling. Further study is required to determine the number of antennas required to reliably detect smaller and deeper tumors, particularly for the examination of more realistic breast environments.

In addition to providing reliable tumor detection, CMI effectively localizes the tumor response in three dimensions. As indicated in Table II, the location of the maximum tumor response closely resembles the physical location of the tumor center. The FWHM response, a measure of the physical extent of the tumor, has similar overall volume for both systems, and is only $10 \%$ larger than the actual volume of the 6-mm-diameter spherical tumor. The FWHM response is influenced by the physical span and configuration of the synthetic antenna array. For the planar system, the array span is similar in the $x$ and $y$ directions, resulting in similar FWHM tumor responses for the $x$ and $y$ directions. For the cylindrical system, the antennas encircle the breast in the $x-y$ plane. The tumor is illuminated from all angles in this plane, so a more localized response is obtained in this plane compared with the planar system. However, the antenna feed locations span only $4 \mathrm{~cm}$ along the cylinder axis, resulting in a less localized response in the third dimension than that achieved with the planar system.

To test the influence of array span and tumor location, images are reconstructed with subsets of the cylindrical array. When the span is decreased to $3 \mathrm{~cm}$ [rows 2-8, Fig. 2(b)], the FWHM tumor response along the $\mathrm{z}$ direction increases to $14 \mathrm{~mm}$. With a span of $2 \mathrm{~cm}$ [rows 3-7, Fig. 2(b)], a FWHM response of 18 $\mathrm{mm}$ is obtained. No change is observed in FWHM response in the $x-y$ plane. The case of a tumor that is not located at the axial center of the antenna array is explored in Fig. 15. As the tumor leaves the axial center of the array, the response becomes asymmetric and clutter increases. However, detection and reasonable localization are maintained. While increasing the physical extent of a given array improves localization, sufficient spatial sampling requires a greater number of antennas. On the other hand, it is of interest to minimize the number antennas and,

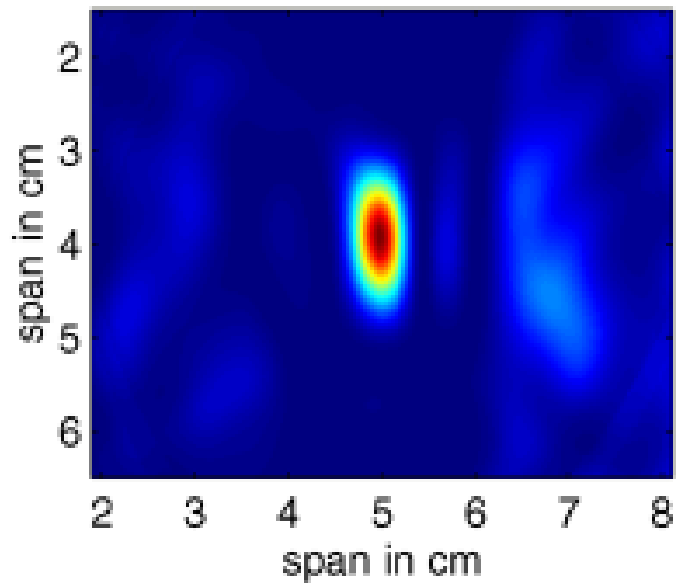

(a)

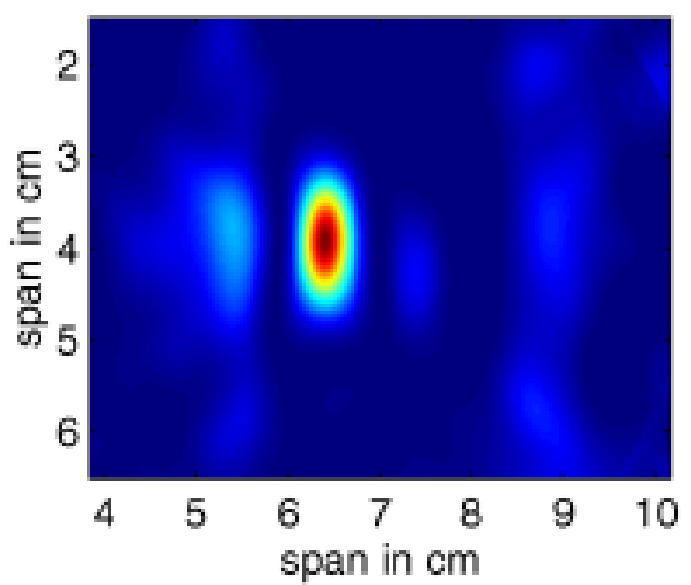

(b)

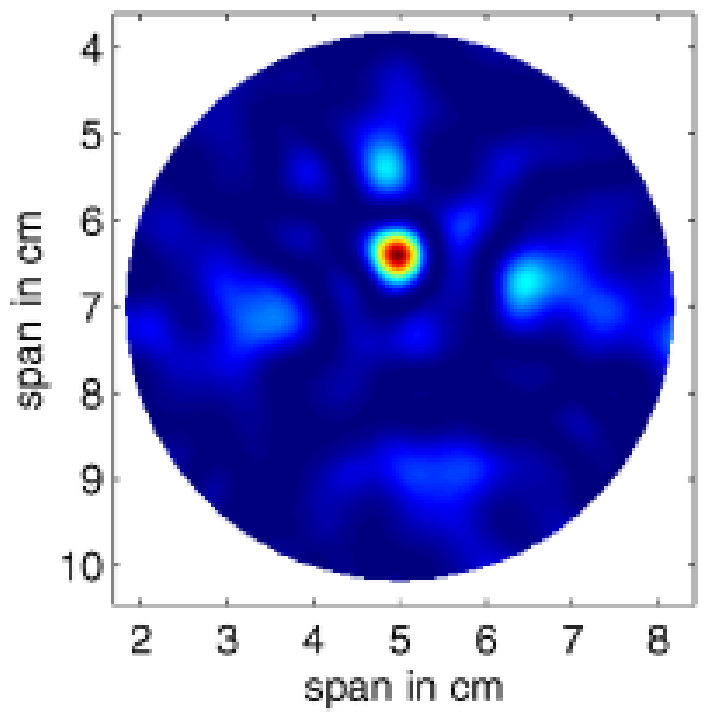

(c)

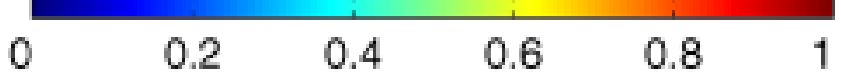

Fig. 12. Three-dimensional reconstructed image, shown in three planes, for the cylindrical system: (a) axial, (b) sagittal, and (c) coronal. The scale for (a)-(c) is shown below (c). 


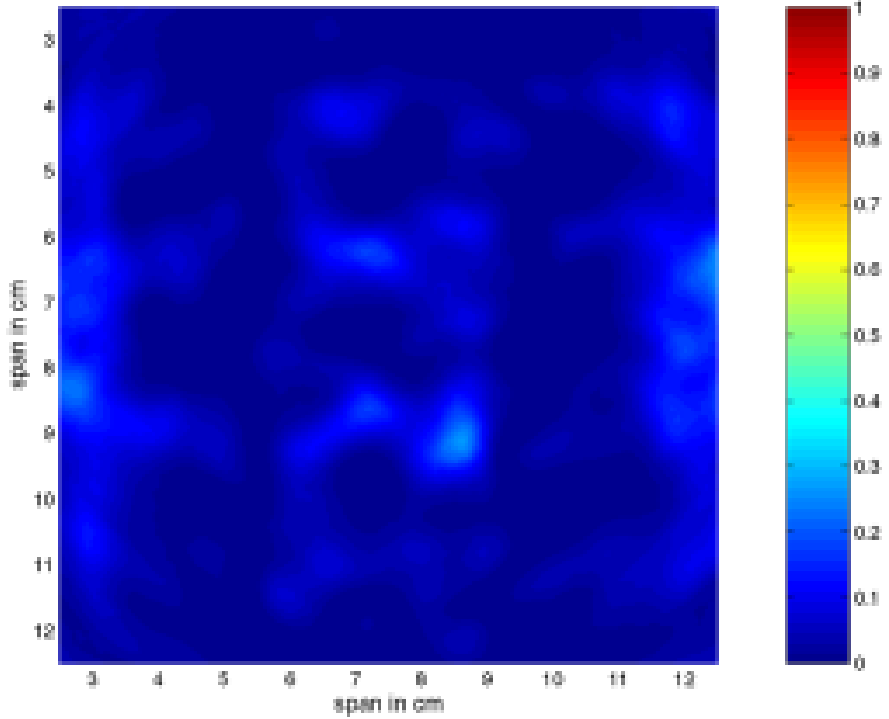

Fig. 13. Reconstructed image for planar system, tumor-free model, coronal view.

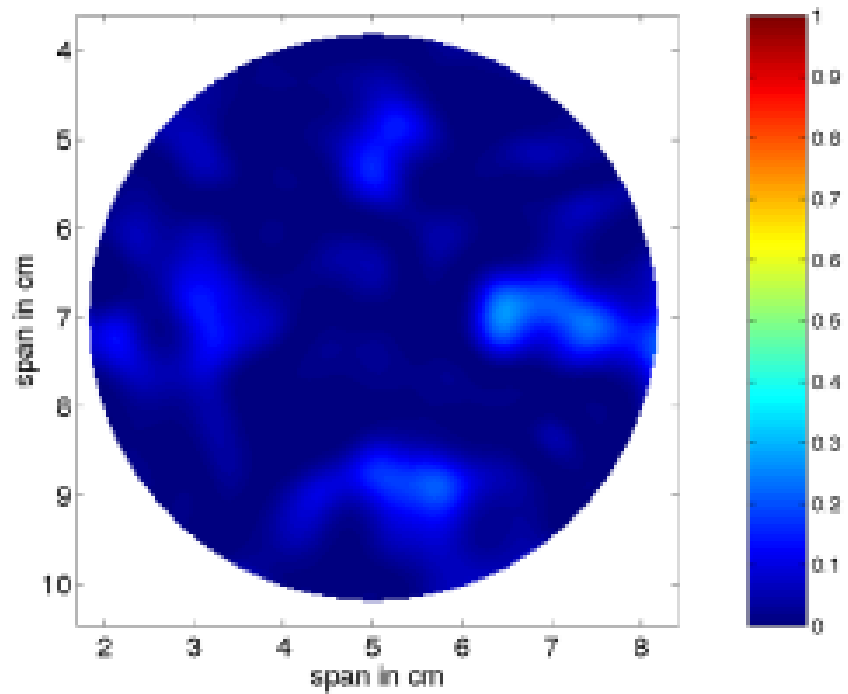

Fig. 14. Reconstructed image for cylindrical system, tumor-free model, coronal view.

therefore, time required for data collection, while maintaining reliable detection and localization of tumors. Further investigation with more realistic models is required to specify the synthetic array configurations required for reliable tumor detection with practical systems.

\section{CONCLUSION}

The results presented in this paper suggest that CMI is a feasible tool for detecting and localizing breast tumors in three dimensions. The image reconstruction algorithms presented require limited a priori information, are effective with both system configurations, and are a simple and rapid way to interrogate the breast for tumors in three dimensions. Both the planar and cylindrical configurations detect tumors with similar within-breast $\mathrm{SC}$ ratios and accurate identification of tumor location. Further
TABLE II

MEASURES APPLIED to IMAGES IN FIGS. 11-14

\begin{tabular}{l|c|c}
\hline \multicolumn{1}{c|}{ Measure } & Planar & Cylindrical \\
\hline Tumor location (mm) & $\begin{array}{c}75,75,59 \\
(75,75,58)\end{array}$ & $\begin{array}{c}64,50,39.3 \\
(64,50,40)\end{array}$ \\
\hline FWHM tumor size: & 123 & 127 \\
- Volume (mm $\left.{ }^{3}\right)$ & Extent (mm) & $\mathrm{x}=8.0, \mathrm{y}=6.5, \mathrm{z}=4.5 \mathrm{x}=4.25, \mathrm{y}=4.25, \mathrm{z}=10$ \\
\hline $\begin{array}{l}\text { Signal-to-clutter ratio: } \\
\text { - Within breast (dB) }\end{array}$ & 3.53 & 4.1 \\
- Between breast (dB) & 21.05 & 13 \\
\hline Clutter statistics & & \\
- Mean & 0.03 & 0.03 \\
- Standard deviation & 0.04 & 0.04 \\
- Number of pixels & 542854 & 10063168 \\
\hline
\end{tabular}

TABLE III

MEASURES APPLIED to IMAGES RECONSTRUCTED With 25 ANTENNAS

\begin{tabular}{l|c|c}
\hline \multicolumn{1}{c|}{ Measure } & Planar & Cylindrical \\
\hline $\begin{array}{l}\text { FWHM tumor size: } \\
\text { - Volume }\left(\mathrm{mm}^{3}\right)\end{array}$ & 109 & 110 \\
- Extent $(\mathrm{mm})$ & $\mathrm{x}=8.0, \mathrm{y}=6.5, \mathrm{z}=4.0$ & $\mathrm{x}=4.5, \mathrm{y}=4.5, \mathrm{z}=9.75$ \\
\hline $\begin{array}{l}\text { Signal-to-clutter ratio: } \\
\text { - Within breast (dB) }\end{array}$ & 1.5 & 2.02 \\
- Between breast (dB) & 11.1 & 24 \\
\hline Clutter statistics: & & \\
- Mean & 0.05 & 0.04 \\
- Standard deviation & 0.05 & 0.06 \\
- Number of pixels & 542854 & 10057940 \\
\hline
\end{tabular}

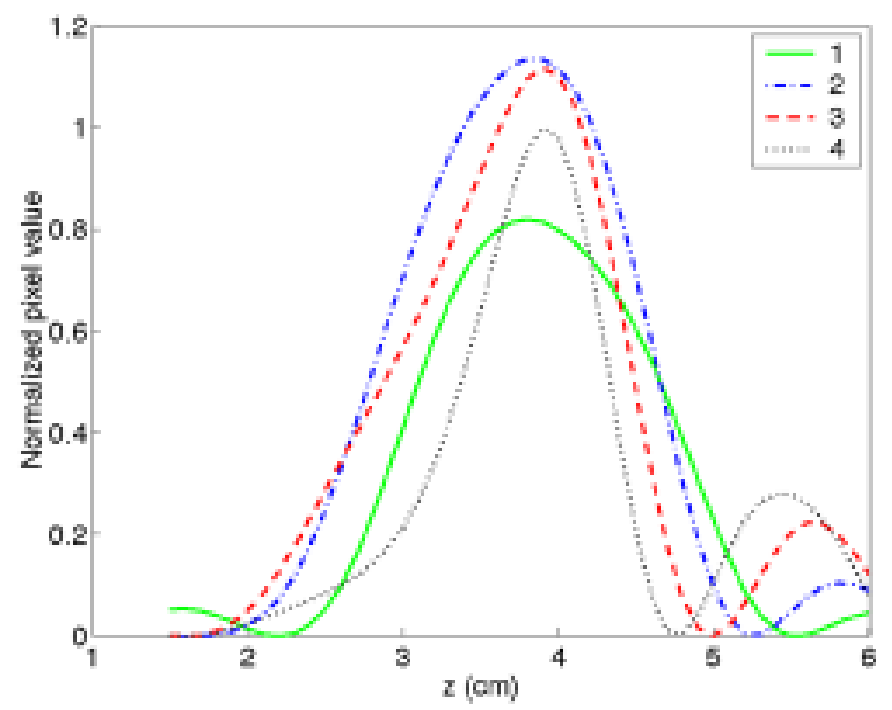

Fig. 15. Tumor response along the $z$ axis for antennas spanning $2 \mathrm{~cm}$. The tumor is located at $z=0.04 \mathrm{~cm}$, and the antennas span 1) $3-5 \mathrm{~cm}$, 2) $2.5-4.5$ $\mathrm{cm}, 3) 2-4 \mathrm{~cm}$, and 4) $1.5-3.5 \mathrm{~cm}$. All tumor responses are normalized to the same maximum as Figs. 12 and 14.

investigation of issues related to practical implementation of CMI will involve anatomically realistic numerical breast models based on high resolution MRI scans, similar to the model presented in two dimensions for the planar configuration case [26], and experimental studies using tissue phantoms. 


\section{ACKNOWLEDGMENT}

The authors would like to acknowledge Cray, Inc. for providing computing resources at the University of Wisconsin-Madison.

\section{REFERENCES}

[1] M. Brown, F. Houn, E. Sickles, and L. Kessler, "Screening mammography in community practice," Amer. J. Roentgen., vol. 165, pp. 1373-1377, Dec. 1995

[2] P. T. Huynh, A. M. Jarolimek, and S. Daye, "The false-negative mammogram," Radiograph., vol. 18, no. 5, pp. 1137-1154, 1998.

[3] J. G. Elmore, M. B. Barton, V. M. Moceri, S. Polk, P. J. Arena, and S. W. Fletcher, "Ten-year risk of false positive screening mammograms and clinical breast examinations," New Eng. J. Med., vol. 338, no. 16, pp. 1089-1096, 1998.

[4] V. P. Jackson, R. E. Hendrick, S. A. Feig, and D. B. Kopans, "Imaging of the radiographically dense breast," Radiology, vol. 188, pp. 297-301, Aug. 1993.

[5] M. Sabel and H. Aichinger, "Recent developments in breast imaging," Phys. Med. Biol., vol. 41, pp. 315-368, March 1996.

[6] S. H. Heywang-Kobrunner, "Nonmammographic breast imaging techniques," Curr. Opinion Radiol., vol. 4, pp. 146-154, Oct. 1992.

[7] A. J. Surowiec, S. S. Stuchly, J. R. Barr, and A. Swarup, "Dielectric properties of breast carcinoma and the surrounding tissues," IEEE Trans. Biomed. Eng., vol. BME-35, pp. 257-263, Apr. 1988

[8] W. T. Joines, Y. Zhang, C. Li, and R. L. Jirtle, "The measured electrical properties of normal and malignant human tissues from 50 to $900 \mathrm{MHz}$," Med. Phys., vol. 21, pp. 547-550, April 1994.

[9] S. S. Chaudhary, R. K. Mishra, A. Swarup, and J. M. Thomas, "Dielectric properties of normal and malignant human breast tissues at radiowave and microwave frequencies," Indian J. Biochem. Biophys., vol. 21, pp. 76-79, February 1984.

[10] S. C. Hagness, K. M. Leininger, J. H. Booske, and M. Okoniewski, "Dielectric characterization of human breast tissue at microwave frequencies," presented at the 2nd World Congr. Microwave and Radio Frequency Processing, Orlando, FL, Apr. 2000.

[11] L. E. Larsen and J. H. Jacobi, Eds., Medical Applications of Microwave Imaging. Piscataway, NJ: IEEE Press, 1986.

[12] S. Caorsi, G. L. Gragnani, and M. P. Pastorino, "Reconstruction of dielectric permittivity distributions in arbitrary 2-D inhomogeneous biological bodies by a multiview microwave numerical method," IEEE Trans. Med. Imag., vol. 12, pp. 232-239, June 1993.

[13] A. E. Souvorov, A. E. Bulyshev, S. Y. Semenov, R. H. Svenson, A. G. Nazarov, Y. E. Sizov, and G. P. Tatsis, "Microwave tomography: A twodimensional Newton iterative scheme," IEEE Trans. Microwave Theory Tech., vol. 46, pp. 1654-1659, Nov. 1998.

[14] W. C. Chew, Advances in Computational Electrodynamics: The Finite-Difference Time-Domain Method, A. Taflove, Ed. Norwood, MA: Artech House, 1998, ch. 12.

[15] A. Franchois, A. Joisel, C. Pichot, and J. C. Bolomey, "Quantitative microwave imaging with a $2.45-\mathrm{GHz}$ planar microwave camera," IEEE Trans. Med. Imag., vol. 17, pp. 550-561, Aug. 1998

[16] M. Bertero, M. Miyakawa, P. Boccacci, F. Conte, K. Orikasa, and M. Furutani, "Image restoration in chirp-pulse microwave CT (CP-MCT)," IEEE Trans. Biomed. Eng., vol. 47, pp. 690-699, May 2000.

[17] P. M. Meaney, K. D. Paulsen, J. T. Chang, M. W. Fanning, and A Hartov, "Nonactive antenna compensation for fixed-array microwave imaging_Part II: Imaging results," IEEE Trans. Med. Imag., vol. 18, pp. 508-518, June 1999.

[18] P. M. Meaney, M. W. Fanning, D. Li, S. P. Poplack, and K. D. Paulsen, "A clinical prototype for active microwave imaging of the breast," IEEE Trans. Microwave Theory Tech., vol. 48, pp. 1841-1853, Nov. 2000.

[19] D. J. Daniels, Surface-Penetrating Radar. London, U.K.: Inst. Elect. Eng. Press, 1996.

[20] R. E. Sepponen, "Medical diagnostic microwave scanning apparatus," U.S. Patent 4641 659, Feb. 10, 1987.
[21] A. W. Preece, H. Johnson, F. L. Green, and M. P. Robinson, "Dielectric imaging for localization and detection of breast tumors," in IEEE MTT-S Int. Microwave Symp. Dig., 1993, pp. 1145-1146.

[22] N. M. Pothecary, C. J. Railton, R. H. Johnson, and A. W. Preece, "FDTD analysis of a noninvasive sensor for the detection of breast tumours," in IEEE MTT-S Int. Microwave Symp. Dig., 1994, pp. 527-530.

[23] S. C. Hagness, A. Taflove, and J. E. Bridges, "Two-dimensional FDTD analysis of a pulsed microwave confocal system for breast cancer detection: fixed-focus and antenna-array sensors," IEEE Trans. Biomed. Eng., vol. 45, pp. 1470-1479, Dec. 1998.

[24] _ "Wideband ultralow reverberation antenna for biological sensing," Electron. Lett., vol. 33, no. 19, pp. 1594-1595, 1997.

[25] _ - "Three-dimensional FDTD analysis of a pulsed microwave confocal system for breast cancer detection: design of an antenna-array element," IEEE Trans. Antennas Propagat., vol. 47, pp. 783-791, May 1999.

[26] X. Li and S. C. Hagness, "A confocal microwave imaging algorithm for breast cancer detection," IEEE Microwave Wireless Comp. Lett., vol. 11, pp. 130-132, Mar. 2001.

[27] E. Fear and M. Stuchly, "Microwave system for breast tumor detection," IEEE Microwave Guided Wave Lett., vol. 9, pp. 470-472, Nov. 1999.

[28] E. C. Fear and M. A. Stuchly, "Microwave detection of breast cancer," IEEE Trans. Microwave Theory Tech., vol. 48, pp. 1854-1863, Nov. 2000.

[29] - "Microwave detection of breast tumors: Comparison of skin subtraction algorithms," Proc. SPIE, vol. 4129, pp. 207-217, 2000.

[30] A. Taflove and S. C. Hagness, Computational Electrodynamics: The Finite-Difference Time-Domain Method, 2nd ed. Norwood, MA: Artech House, 2000.

[31] J.-P. Berenger, "A perfectly matched layer for the absorption of electromagnetic waves," J. Comp. Phys., vol. 114, pp. 185-200, 1994.

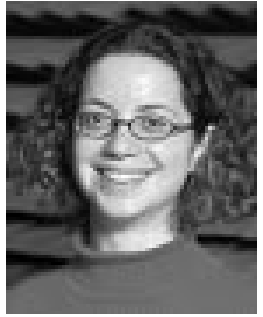

Elise C. Fear (S'98-M'02) received the B.A.Sc. degree in systems design engineering from the University of Waterloo, Waterloo, ON, Canada, in 1995, the M.A.Sc. degree in electrical engineering from the University of Victoria UVic), Victoria, BC, Canada, in 1997, and the Ph.D. degree in electrical engineering also from UVic in 2001.

From 2001-2002, she was a Natural Sciences and Engineering Research Council of Canada (NSERC) Postdoctoral Fellow in electrical engineering at the University of Calgary, Calgary, AB, Canada. She joined the same department as an Assistant Professor in July 2002. Her research interests involve the interaction of electromagnetic fields with living systems, including the interaction of low-frequency fields with biological cells and microwave breast cancer detection.

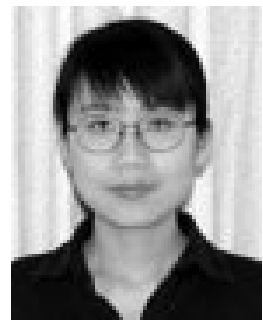

Xu Li (S'99) was born in Beijing, China, on May 13, 1975. She received the B.S. degree in biomedical engineering from Tsinghua University, Beijing, China, and the M.S. degree in biomedical engineering from the University of Wisconsin-Madison, in 1998 and 2000, respectively. Currently she is working towards the Ph.D. degree in the Department of Electrical and Computer Engineering at the University of Wisconsin-Madison

Her research interests include microwave imaging techniques for breast cancer detection, FDTD computational electromagnetics modeling, signal processing and image reconstruction techniques, and ultra-wideband antennas.

Ms. Li was the recipient of a Wisconsin Alumni Research Foundation Graduate Fellowship from the University of Wisconsin-Madison in 1998 


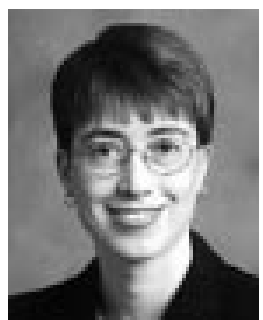

Susan C. Hagness (S'91-M'98) received the B.S. degree with highest honors and the Ph.D. degree in electrical engineering from Northwestern University, Evanston, IL, in 1993 and 1998, respectively.

Since August 1998, she has been with the University of Wisconsin-Madison, where she is currently an Assistant Professor in the Department of Electrical and Computer Engineering and a Faculty Affiliate of the Department of Biomedical Engineering. Her research interests include computational electromagnetics, FDTD theory, bioelectromagnetics, microwave imaging and antennas for biological and medical applications, photonic microstructures and applications. She co-authored Computational Electrodynamics: The Finite-Difference Time-Domain Method, 2nd. ed. (Boston, MA: Artech House, 2000) with Allen Taflove, and has published over 90 journal and conference papers.

In 2000, Dr. Hagness received the Presidential Early Career Award for Scientists and Engineers (PECASE). She also received the UW ECE Holdridge Excellence in Teaching Award. In 2002, she was named one of the 100 top young innovators in science and engineering in the world by MIT's Technology Review magazine. She also received the Booker Fellowship Award from the U.S. National Committee of the International Union of Radio Science (URSI).

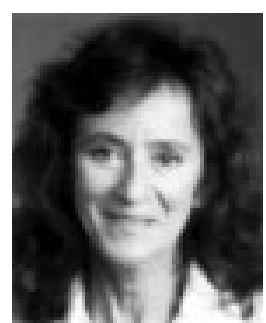

Maria A. Stuchly (S'71-SM'76-F'91) received the M.Sc. degree in electrical engineering from the Warsaw Technical University, Warsaw, Poland, in 1962, and the Ph.D. degree in electrical engineering from the Polish Academy of Sciences, Warsaw, Poland, in 1970.

From 1962 to 1970 , she was with the Warsaw Technical University and the Polish Academy of Sciences. In 1970, she joined the University of Manitoba. In 1975, she joined the Bureau of Radiation and Medical Devices in Health and Welfare, Ottawa, ON, Canada, as a Research Scientist. In 1978, she was associated with the Electrical Engineering Department, University of Ottawa, as an Adjunct Professor. From 1990 to 1991, she was a Funding Director of the Institute of Medical Engineering. In 1992, she joined the University of Victoria, Victoria, BC, Canada, as a Visiting Professor with the Department of Electrical and Computer Engineering. Since January 1994, she has been a Professor and Industrial Research Chairholder funded by the Natural Sciences and Engineering Research Council of Canada, BC, Hydro and Trans Alta Utilities. Her current research interests are in numerical modeling of interactions of electromagnetic fields with the human body and wireless communication antennas. 